\title{
Frédéric Rasera, Des footballeurs au travail. Au cœur d'un club professionnel
}

Marseille, Agone, coll. «L'ordre des choses », 2016

\section{Pierre-Emmanuel Sorignet}

\section{(2) OpenEdition}

\section{Journals}

\section{Édition électronique}

URL : http://journals.openedition.org/travailemploi/8811

DOI : $10.4000 /$ travailemploi.8811

ISSN : 1775-416X

Éditeur

DARES - Ministère du Travail

Édition imprimée

Date de publication : 1 juillet 2018

Pagination : 181-184

ISSN : 0224-4365

Référence électronique

Pierre-Emmanuel Sorignet, «Frédéric Rasera, Des footballeurs au travail. Au cœur d'un club professionnel », Travail et Emploi [En ligne], 155-156 | Juillet-décembre 2018, mis en ligne le 11 juillet 2019, consulté le 23 septembre 2020. URL : http://journals.openedition.org/travailemploi/8811 ; DOI : https://doi.org/ 10.4000/travailemploi.8811 


\section{Des footballeurs au travail. Au cour d'un club professionnel}

Frédéric Rasera

Marseille, Agone, coll. «L'ordre des choses », 2016, 305 p.

Lu par Pierre-Emmanuel Sorignet ${ }^{*}$

Frédéric Rasera présente une ethnographie d'une équipe première d'un club de football évoluant principalement en championnat de ligue 2. Il propose une analyse fine des carrières de footballeurs à travers l'objectivation des contraintes spécifiques générées par l'organisation du travail mise en place par la direction de ce club dans le cadre d'un rapport salarial dans lequel les joueurs n'ont que très peu de marges de négociations. Leur situation de subordination leur est rappelée régulièrement par les dirigeants. L'analyse replace aussi les parcours de ces footballeurs dans les ressorts de leur différenciation sociale. Ce faisant, l'auteur permet d'envisager une sociologie de la vocation tout au long d'un cycle de vie professionnelle marqué par la précarité et la brièveté. À l'inverse d'un grand nombre de travaux qui centrent le propos sur la genèse de la vocation en étudiant la période de formation, l'auteur se concentre sur le quotidien au travail de footballeurs « ordinaires » et analyse les conditions sociales de possibilité du maintien, mais aussi de la recomposition de leur engagement. Plus encore, le choix d'articuler cette perspective à une sociologie des styles de vie, nourrie par la proximité que l'enquêteur a su tisser sur un temps parfois long avec ses enquêtés, s'avère particulièrement convaincant pour exploiter la diversité du matériel recueilli. Enfin, l'auteur privilégie une approche en termes de trajectoire plutôt qu'en termes de carrière, ce qui lui permet d'intégrer dans l'analyse des scènes sociales différentes - l'espace professionnel et l'espace privé - et de ne pas dissocier une temporalité ancrée dans la situation décrite (synchronique) d'une autre qui tient compte de l'histoire des acteurs comme des institutions (diachronique).

L'ouvrage est divisé en sept chapitres. Les cinq premiers s'attachent à déplier les différentes dimensions de l'emploi et du travail des footballeurs enquêtés. Le premier chapitre donne à voir la façon dont les salariés que sont les footballeurs employés par ce club négocient de manière individuelle leur intégration au collectif. L'injonction des dirigeants pour obtenir la réussite sur le terrain exerce une contrainte constante sur les joueurs jusque dans leur vie personnelle. Le chapitre 2 montre que l'incertitude structurelle est au cœur de l'employabilité des footballeurs, dépendants des sélections

* Université de Lausanne. 
incessantes auxquelles ils sont soumis au gré des compétitions. L'originalité de cette vie au travail est d'imbriquer une forte individualisation de la main-d'œuvre que sont les footballeurs inscrits dans un marché où ils sont en constante compétition les uns par rapport aux autres avec l'injonction des dirigeants d'adhérer au collectif. Le chapitre 3 fait intervenir une variable spécifique à ce sport populaire : le souci de représentation, comme l'une des compétences professionnelles à mobiliser dans le travail quotidien. Savoir gérer sa propre communication auprès des médias locaux comme des supporters requiert, de la part de ces footballeurs peu préparés à ce type d'épreuve, un véritable apprentissage pour maîtriser la porosité entre espace privé et espace public local. Le chapitre 4 puis le chapitre 5 font porter la focale sur la gestion du corps au travail, à l'entraînement et dans la compétition. Ce faisant, ils explicitent les formes de domination au travail vécues par les joueurs, mais aussi les résistances qu'ils peuvent déployer, en particulier à travers l'usage tactique de la blessure. Les deux derniers chapitres sont davantage centrés sur la façon dont les normes professionnelles légitimes viennent empiéter sur les styles de vies des joueurs et de leurs familles. Que ce soit dans la tenue d'une bonne « hygiène de vie », dans laquelle la stabilité du couple joue comme condition d'optimisation des performances, ou dans la capacité à démontrer son sens du collectif en nouant des amitiés de vestiaires en dehors du terrain, les joueurs sont traversés par les normes sportives locales, édictées par les dirigeants et encadrants, et relayées par « les anciens » jusque dans leur vie hors-travail.

On perçoit bien ici que la mécanique de la réussite footballistique réside dans l'incorporation combinée d'un style de vie et d'un ethos sportif local, mais aussi dans la capacité à intérioriser un ensemble de comportements où le calcul rationnel est central.

L'ouvrage ouvre de nombreuses pistes de réflexions et d'analogies possibles avec d'autres mondes sportifs ${ }^{1}$, mais aussi avec les professions artistiques ${ }^{2}$. On retiendra ici l'importance du temps dans la démarche ethnographique de l'auteur, lui-même ancien footballeur passé par le centre de formation local, qui connaît certains de ses enquêtés de longue date, condition de son intégration au plus près du quotidien des joueurs.

\section{Un rapport au temps socialement différencié}

Ce faisant, c'est aussi le rapport au temps des enquêtés, comme le produit à la fois de socialisations primaires et d'une socialisation secondaire dans un milieu professionnel marqué par sa propre logique temporelle, qu'il s'agit d'explorer pour comprendre la façon dont les trajectoires des uns et des autres se déroulent sur la durée. Il apparaît ainsi que tous les enquêtés, dont la grande majorité sont issus des classes populaires stabilisées, ne sont pas égaux dans la maîtrise du temps et en particulier de l'anticipation qui conduit les uns à prévoir la sortie du métier en faisant l'inventaire de

1. On pense en particulier au travail de Manuel Schotté (2012) sur les coureurs marocains : La Construction du talent. Sociologie de la domination des coureurs marocains, Paris, Raisons d'agir.

2. Entre autres, Sorignet P.-E. (2010), Danser. Enquête dans les coulisses d'une vocation, Paris, La Découverte et Perrenoud M. (2007), Les Musicos. Enquête sur des musiciens ordinaires, Paris, La Découverte. 
leurs ressources et en recomposant la part vocationnelle de leur investissement, et les autres à réagir dans l'urgence, au coup par coup, se condamnant pour quelques-uns à une plus grande fragilisation de leur statut social. Mettre au jour une corrélation entre origine sociale (ici dans le sens de fraction de classe) et dispositions temporelles, ou, pour le dire autrement, un « espace social des rapports au temps ${ }^{3} »$, incite à étudier la façon dont les ressources sociales participent à la modélisation de l'espace des possibles professionnels jusqu' au moment des reclassements.

Étudier dans la durée ces trajectoires de footballeurs oblige ainsi à réfléchir à l'usage du concept de vocation ${ }^{4}$, à éviter de réifier ces parcours et de les réduire aux moments d'entrée dans le métier, en leur redonnant une épaisseur historique. S'interroger sur le vieillissement dans des métiers dont la force de travail qu'est le corps est destinée à péricliter, en particulier face à d'autres candidats, plus jeunes, mieux formés par rapport aux évolutions de la demande conjointe du marché et des ressources techniques dominantes validées par les coaches, c'est aussi questionner la temporalité de l'enquête.

\section{Des vocations désenchantées?}

L'essentiel des travaux en sociologie de la vocation porte sur les dispositions pour entrer dans des univers portés par la croyance dans la dimension inconditionnelle et nécessaire d'en être ${ }^{5}$. Il en est assez peu qui envisagent les conditions de maintien et d'adhésion dans le temps, voire les désillusions de ceux qui disent avoir « sacrifié » plusieurs années de leur vie à une activité marquée par la précarité et par la fragilité des positions prestigieuses occupées à un instant du parcours ${ }^{6}$. L'enquête menée par Frédéric Rasera dans la longue durée permet de saisir des moments de bascule ; pour autant, il n'y réduit pas les parcours des enquêtés et les met en perspective. Les moments de réenchantement peuvent succéder au désenchantement lié à la difficulté à retrouver un emploi, à une blessure qui éloigne des terrains ou à la lassitude éprouvée au moment de se remettre en jeu dans des procédures marquées par la confrontation in vivo avec des nouveaux entrants plus jeunes, plus motivés et plus dociles, face à des dirigeants obsédés par la performance de l'équipe et des joueurs.

Penser en termes de vocations désenchantées, c'est tenir compte des effets du temps sur les aspirations des enquêtés à embrasser une carrière footballistique, des ressources qui sont mobilisées à différentes étapes de celle-ci. C'est aussi considérer la réalité de ce qui est vécu au quotidien : négocier son temps de jeu, savoir valoriser ses performances tout en restant « collectif », maîtriser les représentations publiques

3. Darmon M. (2013), Classes préparatoires. La fabrique d'une jeunesse dominante, Paris, La Découverte.

4. On s'inscrit ici dans les pas des travaux de Charles Suaud (1978), La Vocation, Paris, Éditions de Minuit.

5. Sapiro G. (2007), « La vocation artistique entre don et don de soi », Actes de la recherche en sciences sociales, $\mathrm{n}^{\circ} 168$, pp. 4-11.

6. Suaud C., Viet-Depaule N. (2004), Prêtres et ouvriers. Une double fidélité mise à l'épreuve, 1954-2004, Paris, Karthala. 
de soi, stabiliser sa relation de couple, développer une sociabilité amicale avec les autres joueurs - en somme, se plier à des normes qui sont autant professionnelles que morales. On perçoit aussi que les enquêtés disposent de ressources sociales différenciées pour se maintenir dans un rythme de vie contraignant et qui nécessite que les conditions propices à la poursuite de l'engagement soient optimisées - en particulier, la disponibilité des femmes, dont les plus ajustées à ce style de vie vivent la carrière sportive de leur conjoint comme l'expression d'une petite entreprise collective.

En conclusion, Des footballeurs au travail est une enquête ethnographique remarquable et rare dans la richesse de ses données, jamais présentée sous la forme d'un jargon théorique mais néanmoins ancrée dans une grille interprétative solide. L'ouvrage peut ainsi se lire sous différents registres, du lecteur profane en sciences sociales mais intéressé par la réalité quotidienne des footballeurs, au sociologue qui y retrouvera une rigueur et une enquête de grande qualité scientifique. 